\title{
INTRACRANIAL TUMOURS
}

\section{A Clinical Review}

\author{
By C. Worster-Drought, M.A., M.D., F.R.C.P. \\ Physician to the West End Hospital for Diseases of the Nervous System and to the Metropolitan Hospital
}

From the clinical standpoint intracranial tumours can be divided roughly into two main groups : (a) Those that affect a particular region only, c.g. pituitary region (adenoma, craniopharyngioma, chordoma), acoustic nerve (neurinoma), choroid plexus (papilloma), pineal region (pinealoma).

(b) Those that may affect any region of the brain, c.g. various types of glioma, meningioma, angioma, metastatic tumours such as secondary carcinoma, etc.

(a) The first, or regional, group may be considered as follows :

(I) Tumours of the pituitary region. 'Tumours of the pituitary body itself are usually adenomata, cither chromophil, basophil, chromophobe, or mixed. The chromophil adenoma is almost invariably associated with some degree of acromegaly if occurring after puberty and if before puberty, with giantism. Adenoma of the basophil cells, according to Cushing, gives rise to a syndrome characterized by increasing obesity, especially of the face, neck and trunk, sexual dystrophy and, in women, hirsutes of the face and trunk. Chromophobe adenomata may be associated with some degree of hypopituitarism.

Other tumours affecting the pituitary region are the group now known as 'craniopharyngiomata' which comprises all squamous epithelial growths, both cysts and solid formations, arising from the remains of the hypophyseal duct. They include, therefore, the tumours previously known as Rathke's pouch tumours, suprasellar and subsellar pituitary cysts, adamantinomata, ameloblastomata and hypophyseal duct tumours. Their origin is congenital and although symptoms may develop at any age from the first to the seventh decade, they most frequently appear in childhood and adolescence.

Finally, a rare tumour known as ' chordoma' may arise from the remains of the primitive notochord in the basi-sphenoid (clivus Blumenbachii) and invade the pituitary region.

(2) Neurinoma. The acoustic neurinoma or neurofibroma arises from the distal part of the auditory nerve. It is often single and unilateral, but may be bilateral or even associated with a diffuse neurofibromatosis either central or peri- pheral. In diffuse central neurofibromatosis, similar and numerous neurinomata are found on the cranial nerves and spinal nerve roots, especially the cauda equina, and often on cranial nerves such as the third, fifth and twelfth (Wishart's syndrome). In addition numerous subcutaneous neurofibromata may be present.

(3) Choroid plexus papilloma. These rarc choroid plexus tumours represent in general a reproduction of the choroid plexus villi and arc said to originate from the latter. Actually they consist of numerous cauliflower-like villi with a central core of loose fibrous tissue and are rich in blood vessels. They occur in the lateral ventricle and in the roof of the third and fourth ventricles.

(4) Pinealoma. This tumour grows from the epiphysis or pineal body and contains two types of cell in groups, $(i)$ loose meshes of large spherical cells with large nuclei and a finely-granular cytoplasm corresponding to the parenchymatous cells of the pineal body, and (ii) smaller lymphocytelike cells lying in groups in a connective-tissue stroma. When of more primitive type and rapidlygrowing, the tumour is termed a ' malignant pinealoma.'

Pinealomata as they continue to grow exert pressure on neighbouring structures in the midbrain and Sylvian aqueduct causing hydrocephalus by obstruction. The third ventricle may become filled with tumour-tissue and lead to compression of the hypothalamus, infundibulum and pituitary body. In addition to the usual results of increased intracranial tension, paralysis of upward conjugatc deviation of the eyes and failure of vision may occur.

Pineal teratomata-both cystic and solid-may also occur. Some of these teratomata when occurring in boys before puberty are associated with pubertas praecox, in which there is precocity of both mental and physical development-the latter especially of the genitalia and secondary sexual characteristics.

(b) The second main group includes the following :

Based on an acldress to the Dartford Division of the British Medical Association, July 1948. 
(1) Glioma-various types (see classification below).

(2) Meningioma, sometimes termed endothelioma and fibroblastoma which arises from the mesothelial cells of the meninges. The cells are oval and epithelial-like and are arranged in the form of islands separated from one another by strands of connective tissue. The tumour often possesses a capsule and does not invade but merely depresses the brain tissue. Of all intracranial tumours they are the most favourable for surgical removal.

(3) Psammoma. A meningeal tumour containing deposits of lime salts. Bruns classifies as psammomata only those tumours which arise in situations where lime salts are normally present, viz. the pineal body and choroid plexus.

(4) Cholesteatoma. Benign and avascular tumours arising mainly from the pia mater at the base of the brain and in the lateral and fourth ventricles. They also occur in the middle ear. 'Their appearance is that of mother of pearl with a smooth surface and a connective tissue capsule.

(5) Angioma including haemangioma and haemangioblastoma. Vascular tumours which may be arterial, venous or mixed. A haemangioblastoma consists of proliferated blood-vessel cells or angioblasts and occurs chiefly in the cerebellum. It is often associated with cystic formations, which may also be found in the kidneys, pancreas and suprarenal body (Lindau's disease).

(6) Congenital tumours (dermoid and teratoma). The dermoid is a sharply defined tumour or cyst arising from displaced embryonic skin cells. Intracranial teratomata are rare and may be associated with other congenital malformations of the brain, spinal cord (spina bifida) or of other parts.

(7) Granulomata (tuberculoma and gumma or syphiloma). Tuberculomata may calcify but occasionally they terminate in tuberculous meningitis.

(8) Metastatic and invasive tumours (carcinoma, sarcoma, hypernephroma, myeloma).

During recent years there appears to have been a considerable increase in the case incidence of primary bronchial carcinoma. Multiple cerebral metastases are especially liable to follow this form of the disease; in fact the patient frequently comes under observation for the first time on account of symptoms of cerebral tumour. The primary growth in the lungs is often so small as to escape detection on clinical and $\mathrm{X}$-ray examination and is revealed only at autopsy. The secondary cerebral tumours are often multiple and occur most frequently in the temporo-sphenoidal and frontal lobes and in the cerebellum.

Metastases of the brain are fairly common in carcinoma of the breast but infrequent in car- cinoma of the bowel. When the latter occurs the lungs also show numerous secondary deposits. Melanotic sarcoma of cutaneous origin may involve both the brain and the bones of the skull, and is usually rapidly fatal.

(9) Primary sarcoma may appear both in the brain and meninges.

(10) Lipoma. 'This type of tumour occasionally occurs within the brain, usually at the base of the skull or above the corpus callosum.

(I I) Osteoma and osteochondroma arising from the bones of the skull.

(12) Various cysts. Subarachnoid cysts, hydatid and cysticercus cysts. Also in the lateral and third ventricle, cysts may originate from the pia mater and choroid plexus.

\section{Glassification of Gliomata}

Bailey and Cushing, in 1926, classified the gliomata as follows :

(I) Astrocytoma. 'The ' glioma durum ' of earlier writers, constituting about 40 per cent. of classified gliomata. The tumour is relatively avascular, hard to the touch and tends to become cystic. Microscopically it consists of cytoplasmic and fibrillary astrocytes, the processes of which form a network. As the tumour grows very slowly, it is relatively benign and patients may survive for long periods even when surgical removal is in complete. Astrocytomata may develop in almost? any part of the central nervous system and they occasionally undergo calcification.

(2) Glioblastoma multiforme or spongioblastoma multiforme. A very malignant tumour occurring almost without exception in the cerebral hemispheres of adults and representing the gliosarcoma of older writers. It grows very rapidly, and its average course is seldom longer than twelve months. As the tumour tends to undergo extensive degenerative changes it sometimes is difficult to classify histologically. Microscopically, it is composed of polymorphic neuroglial cells ; most of the cells are of. bipolar, fusiform shape which cause the growth to resemble a spindle-celled sarcoma.

(3) Medulloblastoma. This is a soft vascular tumour usually occurring in children, and especially originating in the anterior part of the roof of the fourth ventricle in the region of the vermis of the cerebellum. In this situation it is rapidly growing and highly malignant. The tumour has also been found in the cerebrum both of children and adults; in the latter it is sometimes well

1 'A Classification of Tumours of the Glioma Group on a histogenic basis' by Percival Bailey and Harvey Cushing, 1926 and 'Intracranial Tumours' by P. Bailey, I933. 


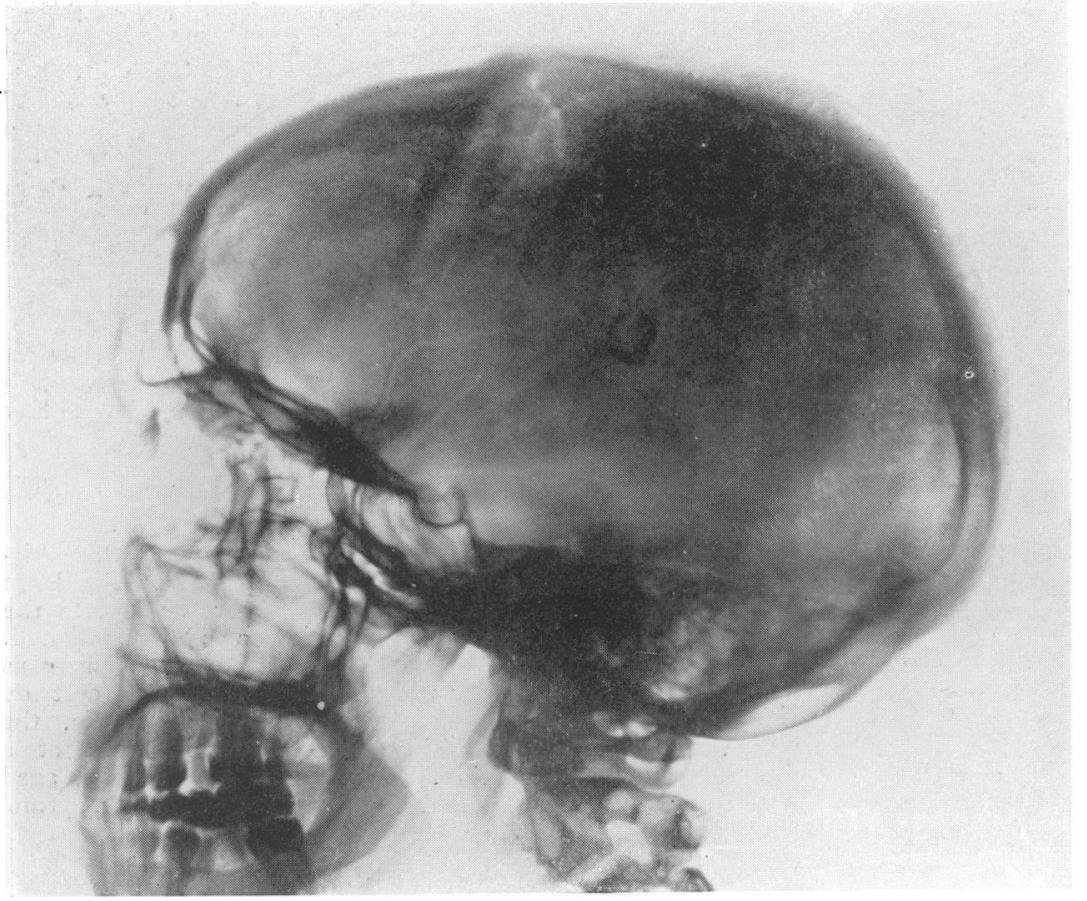

FIG. I.-Calcifying Meningioma. Superficially situated in lower part of right parietal region and eroding the overlying bone. The patient was a woman, aged 22, with focal epilepsy of three years' duration and the tumour was successfully removed by operation.

FIG. 2.-Calcifying Astrocytoma in left frontparietal region. The linear shadows seen in the region indicated suggest that the lesion is a calcified angioma but this diagnosis was excluded by arteriography. The patient was a man, aged 29, with right-sided hemiparesis and focal epilepsy of four years' duration. The greater part of the tumour was removed but recurred two years later.

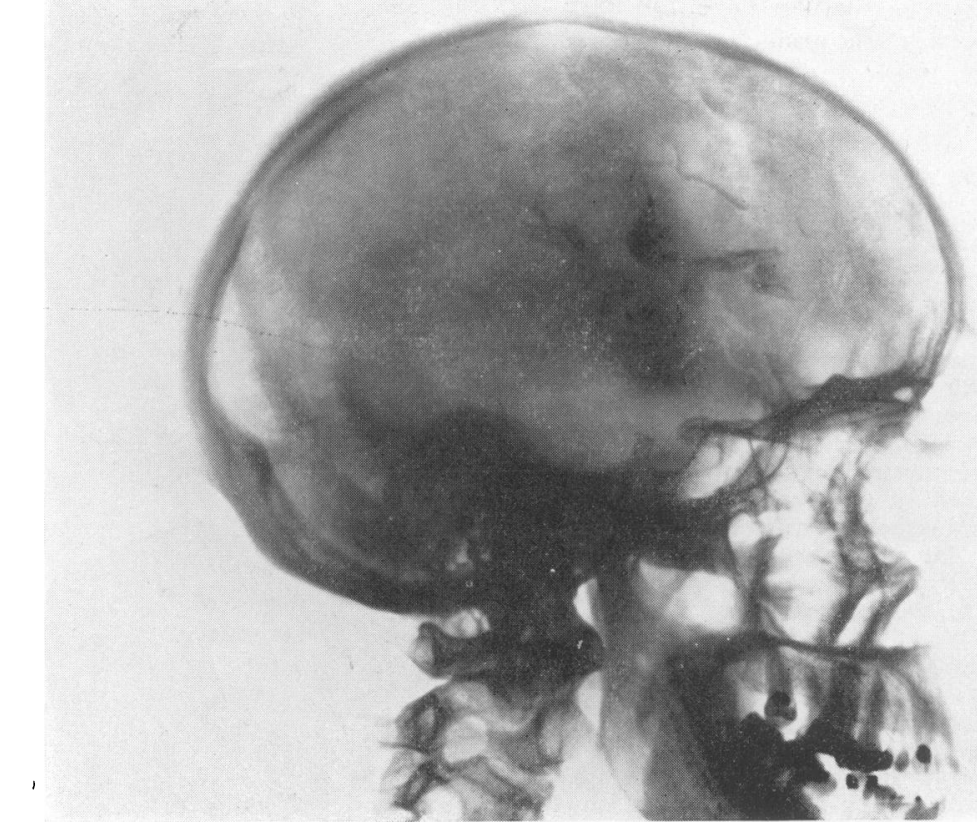




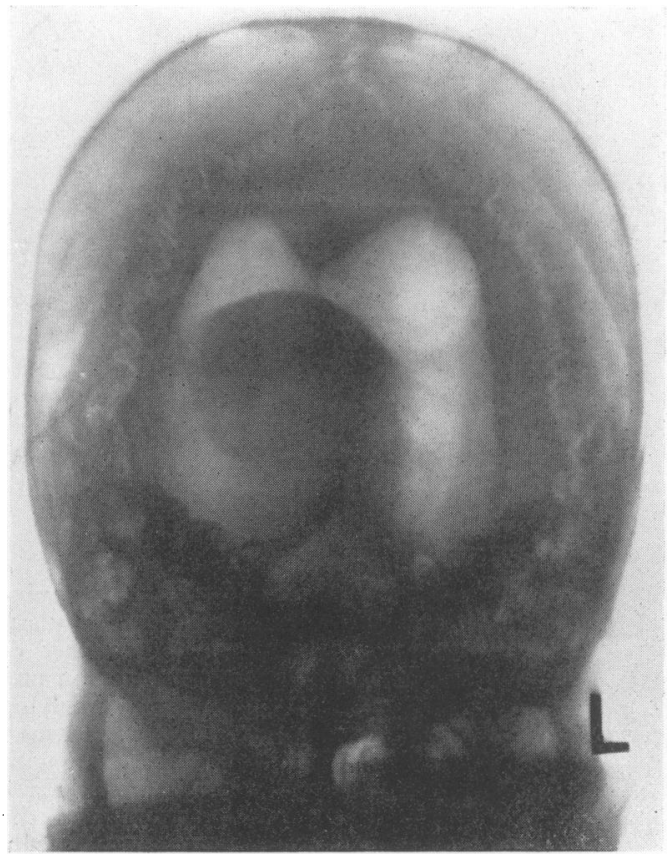

Frg. 3.-Craniopharyngionà (Suprasellat Cyst). Airventriculogram in Townes-Twining position. The large suprasellar cyst is seen projecting upwards and to the right and both lateral ventrieles are considerably dilated. Sixty-four cc. of yellow fluid containing numerous cholesterol crystals were removed from the cyst by trans-ventricular tapping under local anaesthesia, the craniopharyngioma being successfully removed at a second-stage operation. The patient was a man, aged 40, with headache and failing vision of seven years' duration.

Fig. 4. - Medulloblastoma involving the Corpus Callosum. Air-ventriculogram showing a large tumour situated in the midline. The dilated anterior horn of the lateral ventricle is seen in front of the tumour and the somewhat dilated body of the ventricle behind. The patient was a man, aged 48 , with a two years' history of epileptiform attacks and more recent mental confusion; the case proved fatal, the histological diagnosis of the tumour being made post-mortem. 


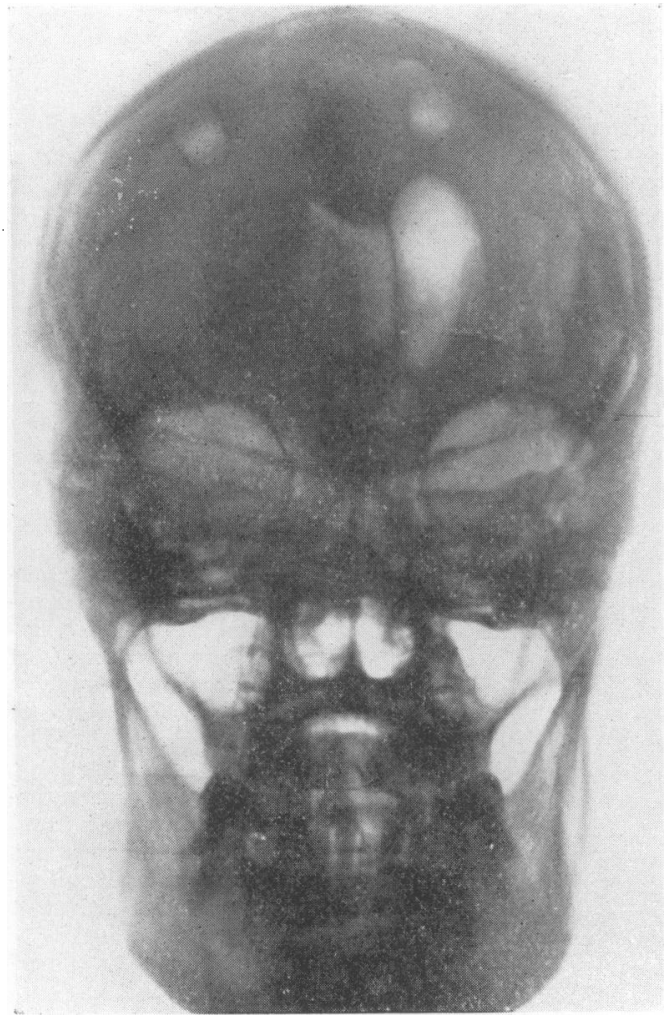

Frg. 5.-Spongioblastoma Multiforme involving right thalamus. Air-ventriculogram showing displacement of ventricular system to left with elevation of floor of right lateral ventricle. In addition, the descending horn of the right lateral ventricle was obliterated and there was an irregular filling defect of third ventricle indicating an extension of the tumour into the hypothalamus. The case was that of a man, aged 45 , and the condition proved fatal.

FIG. 6.-Spongioblastoma Multiforme involving basal ganglia on right side and obliterating the right lateral ventricle. Air-encephalogram showing obliteration of right lateral ventricle by tumour with displacement of the dilated left lateral ventricle and the upper part of the third ventricle to the left. The case was that of a man, aged 69 , which proved fatal, operation not being advised. 


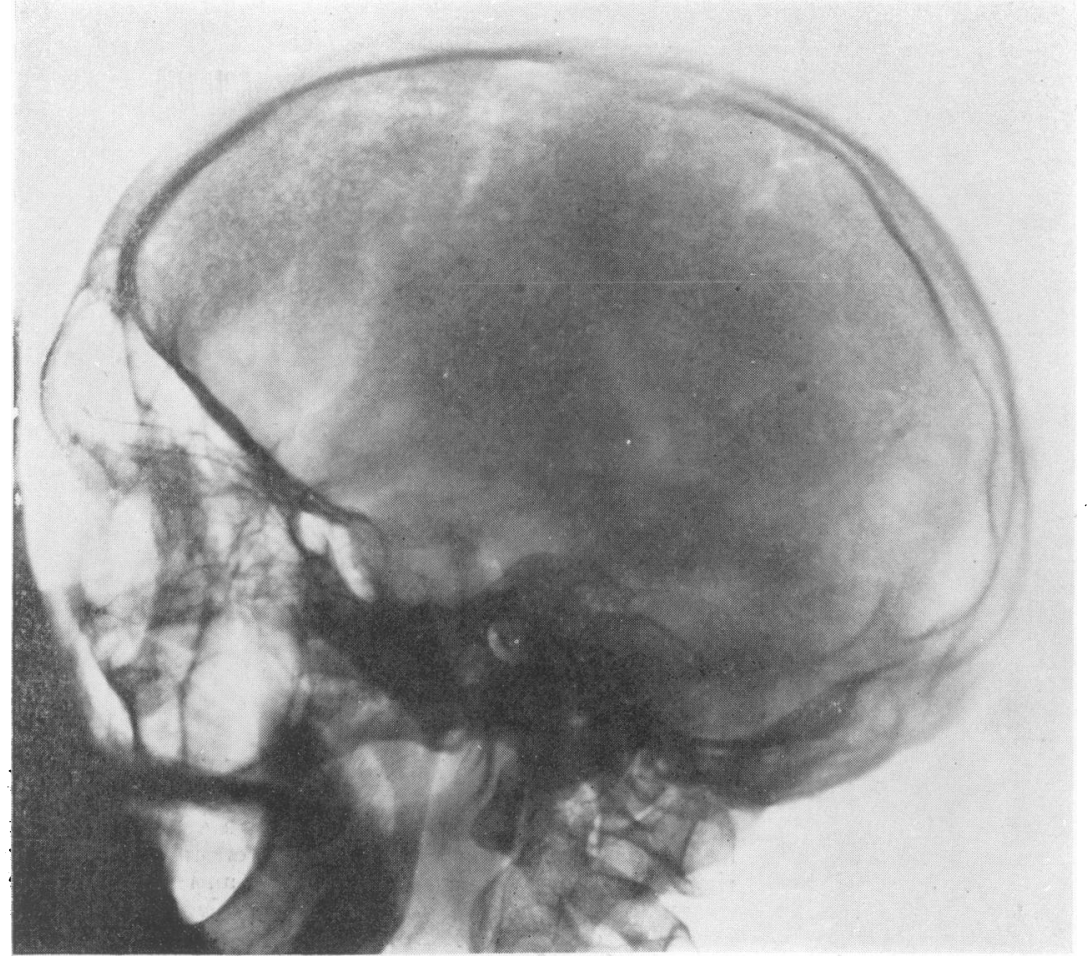

Fig. 7.-Arterio-Venous Angioma of left parietooccipital region. Skiagram showing density of affected area owing to partial calcification of the angioma. The patient, a man aged 33 , had suffered from rightsided hemiparesis and hemianopia since the age of four years and epileptiform attacks for two years.

FIG. 8.-Angiogram of Arterio-Venous Angioma. The same case as illustrated in Fig. 7. The left common carotid artery was ligatured to minimize the risk of intracranial haemorrhage from the angioma; the operation resulted in considerable reduction in frequency of the epileptiform attacks. 
encapsulated and may attain an enormous size. Microscopically the growth is composed of small cells, poor in cytoplasm and with hyperchromatic nuclei; the cells tend to group themselves in the form of rosettes.

(4) Astroblastoma usually occurs in the cerebral hemispheres of adults. It is relatively slowly growing and tends to become cystic. The tumour is rich in blood vessels around which the cells, precursors of astrocytes, are grouped in the form of palisades.

From the point of view of classification, the astroblastomata do not constitute a very well defined group of tumours, as they represent a tumour intermediate between the astrocytoma and the glioblastoma ; consequently, some examples show characteristics of glioblastoma multiforme. In general, however, they are more slowly growing and less malignant than the glioblastomata.

(5) Sponglioblastoma or glioblastoma polare. A neoplasm composed mainly of unipolar spongioblasts extending in parallel rows. It is relatively avascular, slowly growing and benign but is liable to occur in comparatively inaccessible parts of the brain, especially in the mid-line of the brain from the optic chiasma in front to the medulla posteriorly.

(6) Oligodendroglioma. This tumour occurs almost exclusively in the sub-cortical white matter of the cerebral hemisphere of adults, especially in the fifth decade of life. It is very cellular, slowly growing and consists of cells with little cytoplasm with eosinophilic granules. Areas of calcification may develop which show on X-ray examination. The tumour resembles a medulloblastoma but differs from it in that the nuclei are spherical and rich in chromatin.

(7) Ependymoma is a comparatively benign tumour which occurs along the walls of the ventricles, especially the fourth ventricle. In the latter situation it extends into the cerebellum and is usually non-cystic. As the neoplasm grows only very slowly, the patient may survive long after decompression. It originates from ependymal cells and, microscopically, is composed of ependymoblasts.

(8) Ganglioneuroma or neurocytoma. A pathological curiosity. The tumour is very cellular, consists of numerous ganglion and glial cells and is closely related to the oligodendroglioma. It occurs principally in the tuber cinereum, medulla and cerebellum and may undergo calcification. These tumours are relatively benign.

(9) Neuro-epithelioma or medullo-epithelioma. A highly malignant tumour composed of embryonic nerve cells. They are rare in the brain itself but more frequent in the spinal cord and in the retina. In the brain they occur more specially in children, arising from the roof and floor-plates of the third and fourth ventricles. The characteristic microscopical feature is the presence of canals surrounded by primitive spongioblasts in the form of rosettes.

\section{Glinical Aspects}

From the clinical standpoint we may consider the symptoms of intracranial tumour also under two headings :

(a) General symptoms and signs owing to the presence of a tumour within the cranial cavity.

(b) Localizing signs according to the situation of the tumour.

(a) General symptoms and signs: These include the symptoms of headache ; occasional vomiting without relation to meals; papilloedema or swelling of the optic disc; and occasionally epileptiform attacks. Headache is by no means constant and may sometimes be absent in pituitary tumours, frontal tumours, etc. Vomiting is also variable and more liable to occur as a later symptom with increasing intracranial tension. Papilloedema is most frequent in cerebellar tumours, next in frontal and basal tumours; it may be absent in tumours of the parietal and mid-brain regions. Papilloedema of more than four dioptres is an indication for decompression in order to save vision. Epileptiform attacks are an occasional symptom and are more liable to be met with in association with cortical and subcortical tumours especially of the parietal and frontal lobes or in the more severe degrees of intracranial tension.

(b) Localizing signs : These vary according to the situation of the neoplasm. In parietal lobe tumour there is a gradually increasing contralateral hemiplegia, and if the lesion is on the left side, probably aphasia in addition. Focal epilepsy affecting an arm or a leg may also occur. Occipital lobe tumours will give rise to visual field defectscontralateral homonymous hemianopia, etc. Frontal lobe tumours are often associated with confusion, amnesia, a grasp-reflex, and possibly with some aphasia and contralateral hemiplegic signs. Mid-brain tumours cause pupillary changes and oculomotor defects, occasionally emotional disturbances and euphoria. Cerebellar neoplasms are indicated by nystagmus, ataxia, incoordination of the homolateral limbs and often by dysarthria.

Tumours of the pituitary region result in disturbances of the visual fields (e.g. bilateral temporal hemianopia) and occasionally dystrophic endocrine syndromes.

Acromegaly always indicates an eosinophil adenoma of the anterior pituitary lobe. The same observation may be made with regard to most cases of giantism, especially those occurring before puberty. 
Craniopharyngioma enlarging before puberty usually gives rise to some degree of dyspituitarism. This is sometimes of the Fröhlich type-dwarfism, obesity and genital dystrophy. If the hypothalamus is also involved, the hypopituitarism may be of the Levi-Lorain type (infantilism, etc. without obesity). When symptoms of craniopharyngioma do not appear until the third to sixth decade, growth and development may have been normal ; the initial symptom is then usually some visual disturbance such as hemianopia, papilloedema, or optic atrophy.

Basophil adenoma (Cushing's syndrome) is associated clinically with rapidly developing and often painful obesity especially of the trunk and face, a tendency to kyphosis, amenorrhoea in women and functional impotence in men, hypertrichosis of the face and trunk in females and a dusky appearance of the skin with lineae atrophicae and a raised blood pressure.

Adult hypopituitarism may result from a pituitary adenoma of purely chromophobe type.

The solitary acoustic neurinoma or neurofibroma gives rise to a characteristic clinical syndrome. This consists in nerve deafness on the affected side and often tinnitus. Vertigo, owing to involvement of the vestibular portion of the eighth nerve, may also occur and is occasionally the initial symptom. Vestibular tests (caloric and rotatory) will indicate a lesion of the eighth nerve. Neighbouring cranial nerves are next affected-usually the fifth as shown by paraesthesia or anaesthesia of the cheek. The seventh nerve often escapes paralysis but may suffer irritation as indicated by occasional facial twitching; the sixth nerve may also be affected. Pressure of the tumour upon the neighbouring lobe of the cerebellum leads to nystagmus towards the affected side and ataxia of the homolateral arm and leg. As the tumour enlarges the pons may be compressed giving rise to motor weakness and increased deep reflexes in the contralateral limbs. With increasing intracranial tension, papilloedema usually develops.

\section{Diagnosis}

A provisional diagnosis of intracranial neoplasm is made by careful attention to the symptoms and physical signs mentioned above, especially to the possible presence of papilloedema. For confirmation of the diagnosis we have the following additional investigations :

(I) Cerebrospinal fluid examination. On performing lumbar puncture for the withdrawal of cerebrospinal fluid, the pressure of the latter is often found to be increased (over $180 \mathrm{~mm}$.). Examination of the fluid will usually show no increase from the normal number of cells but an increased protein content, e.g. 0.06 to 0.15 per cent. or more (normal 0.025 to 0.04 per cent.). Occasionally the cerebrospinal fluid is slightly yellowish in colour (Froin syndrome) and may contain fibrin in addition to increased protein. The other constituents remain normal. A gumma is excluded by a negative Wassermann reaction in the blood and cerebrospinal fluid.

(2) X-ray examination. A general thinning of the skull-bones due to increased intracranial tension may be demonstrated, or the bone sometimes shows the appearance of beaten copper owing to pressure of the convolutions producing localized thinning. If the tumour is near the surface of the brain, as in the case of some meningiomas, we may see local erosion of the bone adjacent to the tumour (Fig. I). Also a tumour that has undergone calcification (e.g. meningioma, astrocytoma, oligodendroglioma or tuberculoma) may reveal its presence in the radiogram (Fig. 2).

In acoustic neurinoma, it is possible occasionally to demonstrate erosion of the petrous portion of the temporal bone and dilatation of the internal auditory meatus.

Displacement of the pineal body may assist diagnosis and lateralization of the tumour. In about 60 per cent. of normal individuals the pineal body is calcified and can be demonstrated on X-ray examination; in an antero-posterio view, its displacement to one side suggests neoplasm on the opposite side. Similarly, a calcifiec falx cerebri or a calcified choroid plexus may also be displaced by a cerebral tumour.

In the diagnosis of pituitary tumour radiography is of inestimable value. When the tumour arises within the sella turcica (e.g. chromophil adenoma) the pituitary fossa is seen to be uniformly expanded or 'ballooned.' This finding is the rule in acromegaly. Suprapituitary tumours (craniopharyngioma) tend to depress the sella turcica leading to flattening of the pituitary fossa and often to erosion of the clinoid processes. Extensive destruction of bone in the pituitary region suggests chordoma or a basal meningioma.

Areas of calcification above the sella turcica are sometimes seen in cases of craniopharyngioma.

(3) Air-encephalography and ventriculography. These radiographic methods are of the greatest assistance in the diagnosis and localization of cerebral tumours.

Air-encephalography consists in the removal of cerebrospinal fluid and the introduction of air into the subarachnoid space by means of ordinary lumbar puncture. By regulating the position of the patient's head the air rises in the subarachnoid space and fills the cisterna magna and the cortical subarachnoid space; if the foramina of Magendie and Lushka are patent, the air will also penetrate into the fourth ventricle and lateral 
ventricles. The air is injected only very slowlyabout I cc. per minute-the total amount injected varying between 30 and $65 \mathrm{cc}$. This procedure can be carried out only if the cerebrospinal fluid pressure is within normal limits, that is, not above $180 \mathrm{~mm}$. If the cerebrospinal fluid pressure is above this level, air-encephalography by lumbar puncture is unsafe and one has to resort to airventriculography.

Air-ventriculography. The lateral ventricle is punctured over Keen's point ; cerebrospinal fluid is then withdrawn and replaced by an equal quantity of air by means of a two-way tap joining the needle and syringe. It is preferable to puncture both ventricles as if only one is entered, the cerebrospinal fluid in the other ventricle cannot be completely replaced by air and considerable manipulation of the head is required to enable any air to pass from the punctured lateral ventricle into that of the opposite side. According to the changes in symmetry of the ventricles on subsequent $\mathrm{X}$-ray examination, one can often lateralize the tumour and occasionally localize it (Figs. 3, 4 and 5 ).

'Ventricular estimation' may occasionally assist. The quantities of fluid removed from each ventricle on puncture are carefully measured and compared. Normally, the amounts are approximately equal on the two sides. Any pronounced difference indicates partial obliteration of that ventricle yielding the smaller quantity of fluid and this is usually on the side the tumour is situated. Not infrequently, it is found impossible to obtain any cerebrospinal fluid from the lateral ventricle of the affected side owing to its obliteration by the tumour (Fig. 6).

(4) Angiography. This method of investigation consists in the injection of a radiographically opaque substance-thorotrast-directly into the carotid artery and at once (within 2 seconds) taking an X-ray photograph of the head from the lateral aspect, the patient having previously been placed in position. The arteries are then seen outlined in the developed X-ray film (arteriogram). Thus, the presence or otherwise of a suspected cerebral angioma may be confirmed (Figs. 7 and 8 ). Arteriography is also of value in the demonstration of aneurisms, especially those on or in the neighbourhood of the arterial circle of Willis. By means of accurate timing ( 4 seconds) the outline of the veins may also be obtained on a subsequent film (venogram) and a purely venous angioma, if present, demonstrated. Also, cerebral tumours other than angiomata can sometimes be outlined.

The usual method of angiography is to expose the common carotid artery in the neck in order to ensure accurate injection. More recently, a percutaneous technique has been developed, the injection being made into the carotid artery through the skin.

(4) Electro-encephalography. The cells of the normal resting cortex produce delicate spontaneous oscillations of bioelectrical potential. These can be detected through the intact skull and scalp by suitable electrodes, valve-amplifiers and a simple ink-writing oscillograph. The normal or so-called ' alpha' rhythm has a frequency of about ro oscillations per second with an amplitude of 60 and 100 microvolts.

It is rarely that any single method of clinical investigation alone can be expected to establish a complete diagnosis, and this observation applies equally to electro-encephalography. The results of the latter must be correlated with those obtained from physical, pathological and radiological examinations. Nevertheless, with the aid of the electro-encephalogram it is often possible to assist in localizing a cerebral tumour. The cells of the latter are electrically inactive, but at the periphery of some tumours, e.g. a meningioma, or in a cortex infiltrated by a glioma, abnormally slow or ' delta' waves, as they are termed, from $I$ to 5 per second may be recorded and have a pathological and localizing significance.

\section{Differential Diagnosis}

Other conditions which may simulate intracranial tumour are :

(r) Renal disease with uraemia. Headache and vomiting are frequent symptoms, and even optic neuritis, monoplegia or hemiplegia may be present. The diagnosis is made by careful attention to the examination of the urine, especially to the presence of casts, the blood pressure, and the urea content of the blood and the cerebrospinal fluid.

(2) Meningitis (various forms). The presence of neck rigidity, Kernig's sign and great increase in the number of cells in the cerebrospinal fluid (which may be turbid) usually give the diagnosis.

(3) General paresis (Dementia paralytica, G.P.I.). Cerebral tumour may be simulated by this condition, especially if mental confusion, vomiting, optic neuritis, and some form of muscular weakness is present. The diagnosis is made on the positive W.R. in the blood and cerebrospinal fluid and in the latter a Lange curve of 'paretic' type.

(4) Encephalitis and encephalo-myelitis. Changes in the fundus are usually absent and after the first few days the cerebrospinal fluid is usually found to be normal, that is, with no increase in cells or in the protein content. In doubtful cases, X-ray examination and ventriculography will assist in excluding intracranial tumour.

(5) Cerebral arteriopathies. The differential diagnosis from cerebral tumour is often difficult 
especially if signs of a focal lesion exist (e.g. hemiparesis or hemianopia). The difficulty is increased by the facts that $(i)$ in cerebral arteriopathy some increase in the protein content (up to 0.08 per cent. or more) of the cerebrospinal fluid is often found and (ii) a cerebral tumour may coexist with arteriosclerosis. Although an optic neuritis may occur in the latter, changes in the disc itself are usually absent. Retinal arteriosclerosis is nearly always present, the state of the retinal vessels being the best guide to the condition of the cerebral arteries. The presence of a high blood pressure favours arteriosclerosis; on the other hand, in the presence of extensive atheroma of the cerebral vessels the blood pressure may often be quite low. A history of transient palsies or of a sudden onset is more indicative of arteriopathy. In some cases it is necessary to keep the patient under observation for some time in order to exclude cerebral tumour.

(6) Cerebral abscess. The diagnosis is made mainly on the presence or history of discharge from the ear, sinusitis or other source of infection. The commonest sites are the temporo-sphenoidal lobe and the cerebellum. Papilloedema if present at all is usually slight. The cerebrospinal fluid may show a slight increase in the number of lymphocytes-even a few polymorphonuclear cells-as well as in the protein content. The presence of polymorphs in the cerebrospinal fluid does not necessarily indicate abscess as they may occur in association with some gliomas (e.g. glioblastoma). Also, a leucocytosis in the blood may occasionally be found in cerebral tumour as well as in abscess. In some cases a definite differential diagnosis can only be made by exploration.

\section{Treatment}

In general, if the tumour can be localized, operation should be performed with a view to its possible removal. If extirpation is not practicable, as much as possible of the tumour can be removed and the remainder subjected to diathermy. Subsequently deep X-ray treatment can be applied. In deep-seated tumours, radon seeds have been placed around the tumour with occasional benefit. If the tumour cannot be localized and papilloedema is approaching 4 dioptres, decompression should be carried out, either a right-sided subtemporal decompression or one over the suspected site of the tumour.

In cases of pituitary tumour it is a safe rule not to operate for purely endocrine symptoms.

If operation is impracticable or undesirable for any reason the only resort is deep X-ray treatment.

As a temporary measure for reducing increased intracranial tension, the intravenous injection of 50 to $100 \mathrm{cc}$. of a 50 per cent. solution of sucrose is often successful and has superseded the hypertonic saline ( 15 to 30 per cent.) formerly used. A more slowly acting method of reducing intracranial tension is by the rectal administration of a 25 per cent. solution of magnesium sulphate, according to the quantity the patient will retain, up to a total of $25^{\circ} \mathrm{cc}$.

\title{
RECURRENT UMBILICAL PAIN IN CHILDHOOD
}

With special reference to non-specific mesenteric adenitis

\author{
By JoHn APLey, M.D., M.R.C.P. \\ Department of Child Health, Bristol University
}

Partisanship incurs both rewards and penalties. Of its rewards, the most obvious is increased opportunity for exploitation; of its penalties, the outstanding one is the hindrance of progress in other directions. In many branches of medicine the results of over-emphasis have proved in this way detrimental, and among them is the subject under consideration. The wide variety of causes to which 'umbilical colic' has been attributed, will be illustrated during this discussion. They range between such extremes as 'neuropathic constitution' and ' chronic constipation,' accord- ing to the bias of the observer due to variations both in opportunity and in the method of approach.

The main purpose of this paper is to survey the existing extensive contributions in the light of information derived from cases investigated personally.

\section{Causes of chronic or recurrent abdominal pain in childhood}

As a first step it will be helpful to set out a comprehensive list of possible causes of pain which may be localized to the umbilical region. It is 